\title{
ANALYSIS OF HIGH RESOLUTION STELLAR LINE PROFILES
}

\author{
David F. Gray \\ Department of Astronomy \\ Universily of Western ontario \\ London, Ontario, Canada
}

1. Introduction

High resolution implies that we obtain some information on spectral line shapes. In late-type stars, we need to measure velocities of a few $\mathrm{km} / \mathrm{sec}$ to accomplish this. Increasing the spectral resolution and the signal to noise ratio allows us to progress step by step toward deeper physical understanding. The steps we take often lead to good debate and "stimulate" our lives. I am sometimes amused at the urgency we feel to press on to the next step. We rarely seem to pause and enjoy the completion of previous steps. Perhaps this is because we always see shortcomings in completed work. Quite typically one will "discover" the importance of some physical phenomenon (It makes little difference how many others already know about it.), and in his eyes everything done previously becomes wrong because this phenomenon was not included. We used to hear how Milne-Eddington or Schuster-Schwarzschild model atmospheres were inadequate we had to use instead properly computed depth dependence. We used to hear how LTE models were no good - we had to use more detailed physics. Now we talk about line analyses being inadequate because it has not included velocity fields. The curious thing is that we believe that including our pet phenomenon gives the correct models. We ignore all those other phenomena as yet unseen! (Is this a mechanism for maintaining sanity?) I think it really amounts to a statement of what we are able to measure, compute, or understand.

Observationally, I view the situation in steps of "toughness", i.e. how difficult it is to obtain certain types of information from spectral line shapes. Roughly these can be grouped into

1) line widths - typically a half width is measured but we get no information about what causes the broadening.

2) line broadening - where shapes as well as widths are measured and we begin to discern the characteristics of broadening resulting from rotation or macroturbulence.

3) the details - we see line asymmetries or other structure; we measure true central line depths. Questions concerning the physical mechanisms for turbulence can be tackled, $T(\tau)$ derivations become possible, and non-LTE effects can be disentangled. 
Generally, each degree of toughness involves higher spectral resolution and better signal to noise. The detrimental effects of line blends also become more bothersome as we progress to a tougher category.

I will concentrate on the second and third categories of toughness and largely omit the first one. Part of line analysis is knowing how good the data is. We now consider modern capabilities in stellar spectroscopy.

\section{Observational Considerations}

The number of stars available for high resolution studies is restricted in practice by photon rate limitations. Typically $20 \mathrm{~m} / \mathrm{pixel}$ is appropriate for work on latetype stars for a resolution of $-50 \mathrm{~m} \AA(-3 \mathrm{~km} / \mathrm{sec})$. A $20 \mathrm{~m} \AA$ slice is pretty tiny when it comes to building up good photon statistics. Everyone knows that signal to noise goes as $\mathrm{N}^{1 / 2}$ but also recall that the time required to collect a given number of photons varies as the cube of the resolution (or if multielement detectors are used and the size of the field is not of interest, then as the square of the resolution). In the resolution regime I am considering here, even 3-4 magnitude stars begin to look faint. Large telescopes are important for bright star work.

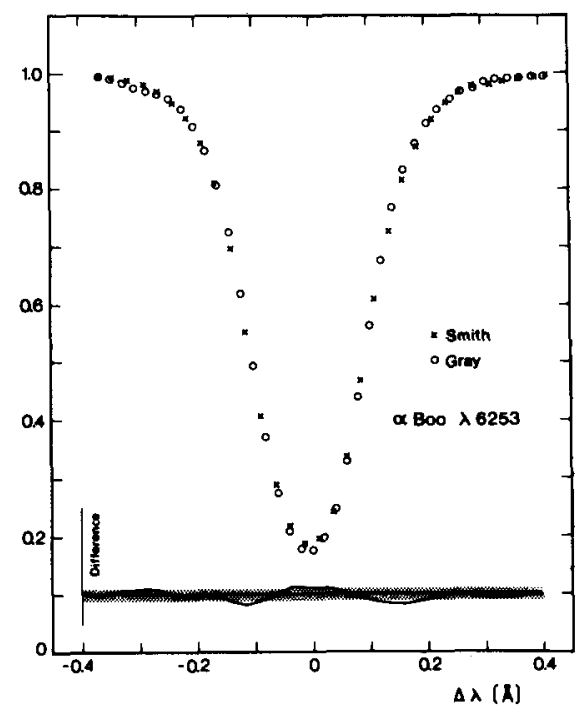

Fig. 1. Two sets of measurements of Fe I $\lambda 6253$ for Arcturus are compared. The differences are plotted on the same scale below the profile. The dotted strip is $\pm 1 \%$ in height.

I have recently compared line measurements made with four totally different sets of equipment in order to see what errors exist with modern measurements. As you know, past comparisons have shown errors of 10-20\% to be common (e.g. Wright et al. 1964, 
Conti and Frost 1977). Fig. 1 shows Fe I $\lambda 6253$ in Arcturus as measured by M. Smith using a Reticon on the McDonald $2.71 \mathrm{~m}$ telescope coude and by myself using a Digicon on the U.W.O. $1.22 \mathrm{~m}$ telescope coude. After allowances for differences in scattered light, the average deviation over $\pm 0.4 \AA$ is $0.7 \%$. Fig. 2 shows a similar comparison

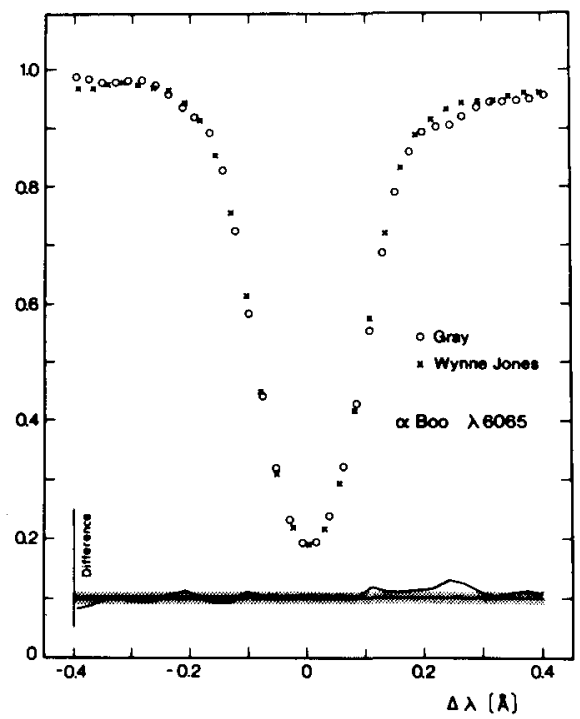

Fig. 2. Measurements of Fe I $\lambda 6065$ for Arcturus are compared.

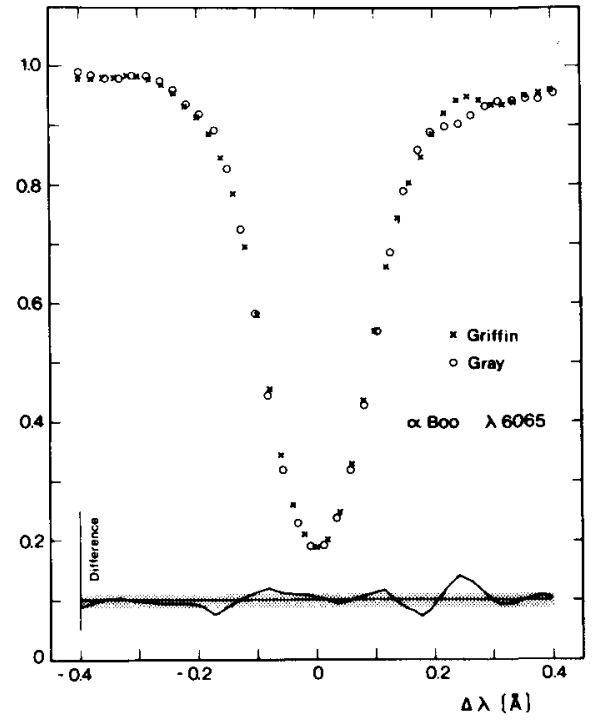

Fig. 3. Heasurements of Fe I $\lambda 6065$ for Arcturus are compared.

for $\mathrm{Fe} I \mathrm{I} 6065$ in Axcturus. The measurements of ivynne-Jones were done using the $2.50 \mathrm{~m}$ Herstmonceux telescope to feed a Michelson interferometer. The average deviation here is $0.8 \%$. Fig. 3 shows a comparison of photographic measurements made by Griffin with mine. The average deviation is 1.0\%. A more detailed discussion of this material is being published in the P.A.S.P.

I conclude that line shapes can currently be measured with errors $\$ 1 \%$. This is still not as good as in solar work, but it is good enough to start doing some physics.

Caution though. We still have a range of up to $5 \%$ in zero-level (scattered light?) differences. This is a crucial point for $T(\tau)$ derivations because there we need line center residual fluxes. Relatively large uncertainties in zero level is one reason why I place the measurements of central line depths in toughness category 3 .

\section{Analysis in the Fourier Domain}

a) Basic Concepts

The general discussion of line analysis in the Fourier domain is available in 
several places (e.g. Gray 1976, 1977, 1978; Smith and Gray 1976) and I will assume that you are somewhat familiar with the process.

First, I wish to show you fig. 4. It is a reconstructed profile - or rather two versions of a reconstructed profile - with slightly different Fourier noise filters. The noise level in the original data is about 28 (not bad by stellar standards). Both filters are reasonable and possible choices. I think you will agree that differences in these reconstructions are uncomfortably large. In principle it is possible to get "better" data, i.e. data with lower noise and higher resolution. But that is costly in observing time and may be impossible in practice. Rather, one can avoid the noise filter problem by performing the analysis in the Fourier domain.

A basic point of what happens is illustrated in fig. 5. Here several common transforms are compared. Clearly, in order to

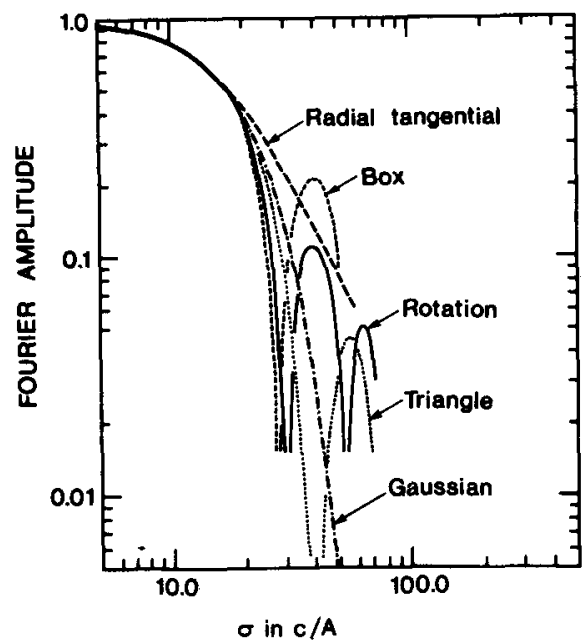

Fig. 5. Thansforms of several commonly encountered functions are compared. Difberences become larger toward high frequencies.

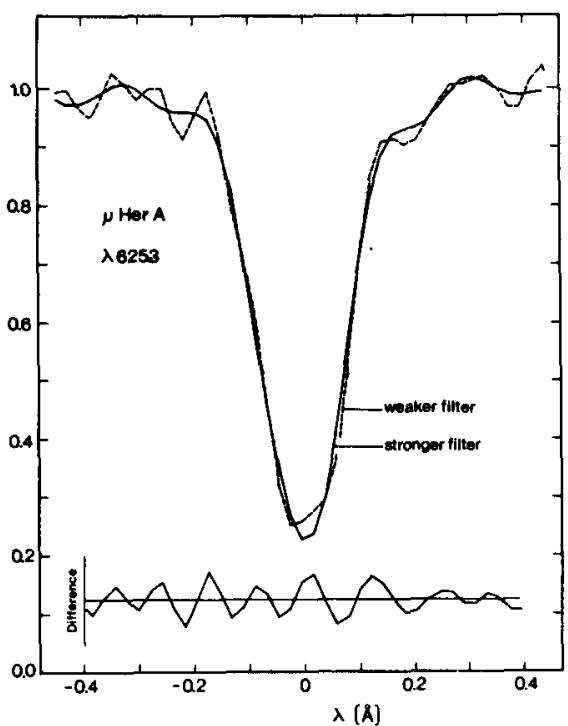

Fig. 4. Two reconstructions of the same set of data showing the effect of the noise filter. The difference is plotted below the profiles.

distinguish between them, it is necessary to measure high enough in frequency and with low enough noise to resolve the differences where they occur.

Fig. 6 emphasizes the signal/noise behavior. For a noise level $n_{t}$, only the top of the main lobe can be seen. As higher photon counts are reached, the high frequency, low amplitude structure is revealed - the details we need to do the science. Notice that the signal/noise is a strong function of $\sigma$ and that the signal is concentrated to limited $\sigma$ bands, e.g. the first sidelobe.

It was with these tools that I attacked the classical problem of separating 
macroturbulence from rotation. If we think way back, we recall that most people used to assume isotropic Gaussian macroturbulence, and it was repeatedly stated that one could not distinguish macroturbulent from rotational broadening except in a statistical sense. Fig. 5 already showed why this was the case and what needed to be done to

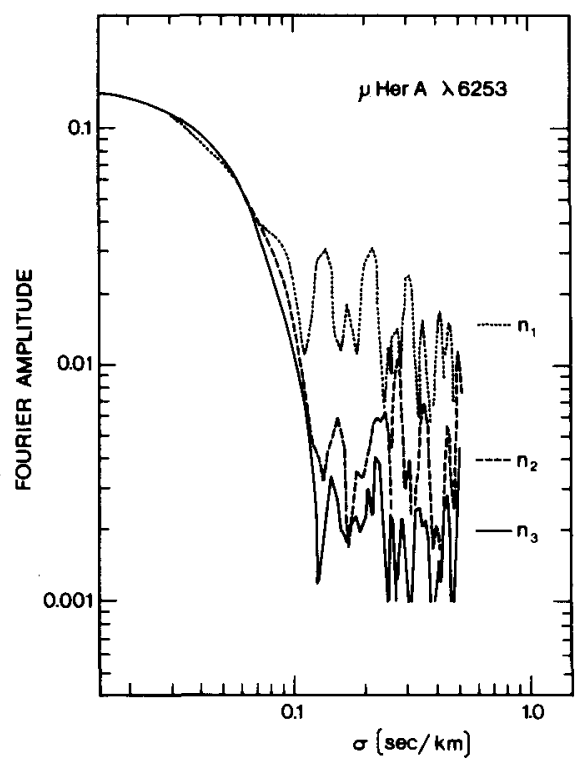

Fig. 6. The noise level can often obscure the information contained in the higher frequencies. solve the problem. At low spectral resolution, the two broadeners look the same. It was necessary to push the observations to the region where they are unique. That has been done. Further, under certain assumptions, it is possible to allocate a certain fraction of the line's macrobroadening to macroturbulence and the remainder to rotation. Obviously this process has meaning only within the context of its basic assumptions - no different than any other operation in science.

b) Convolutions and Disc Integrations Initially I used the convolution approximation to combine the effects of macroturbulence and rotation. This is a valid approximation for some types of macroturbulence and the classical treatment of rotation. But as more involved models come into use, it was necessary to do integrations over the disc (rather costly). The convolution approximation can be used if the functions are independent of position on the disc $(\theta)$. Otherwise integration over the disc cannot be avoided. Still, as a thinking tool, the convolution approximation can be very useful.

Numerical experiments also show that in many applications the full convolution approximation is good to a few per cent. If, for example, we replace the $G(\lambda) \star_{\mathrm{MT}}(\lambda)$ convolution, where $G(\lambda)$ is the rotation profile and $M_{R T}(\lambda)$ is the radial-tangential macroturbulence profile, with a disc integration, we gain the inclusion of limb darkening and a more rigorous treatment of the macroturbulence broadening which is $\theta$ dependent. Assuming equal weighting of radial and tangential components, the disc integrations turn out to give 6 to 88 larger values for $\zeta_{R T}$ than does the convolution formulation. This is almost exclusively a result of limb darkening reducing the broadening of the tangential component.

In addition, we should include the center to limb variation in the $I_{v}$ profile for a complete and proper disc integration. For the solar case, where we can measure this 
change, it is small enough so that neglecting it still gives reasonable results for $\zeta_{\mathrm{RT}}$ and $\mathrm{v} \sin i$ (Gray 1977). For other stars, we do not know the $\theta$ dependence so we cannot put it into the disc integrations even if we want to. We may, of course, postulate a model which gives the $\theta$ dependence and then the complete integration can indeed be done. The postulated model will have to produce a substantial $\theta$ dependence to have much of an effect on the analysis.

Another basic uncertainty exists in the $I_{v}(\theta)$ profile. That is the depth dependence of modeled broadening parameters, e.g. microturbulence dispersion, $\xi$. It is possible to postulate such large gradients in $\xi$ that virtually any shape can be manufactured for $I_{V}(\theta)$ profiles. It quickly becomes a numerical exercise reminiscent of epicycles. Fortunately in many cases the $I_{v}(\theta)$ profile is significantly narrower than the macrobroadening.

c) Precision in Fourier Analysis

Now let me be somewhat more specific in discussing the precision involved once a model has been adopted. We expect quite

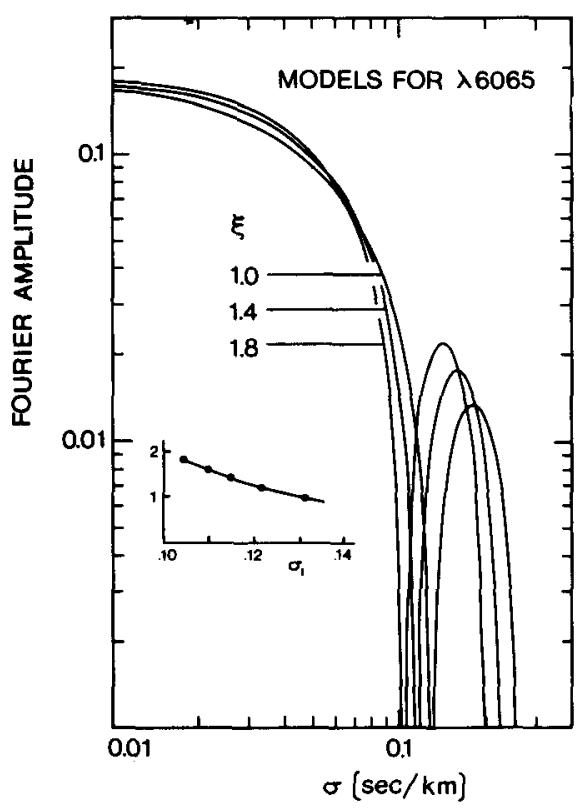

Fig. 1. The position of the first zero in the transform of a saturated line is very sensitive to the degree of saturation. The insert graph shows $\xi(\mathrm{km} / \mathrm{sec})$ as a function of the position of the birst zero $(\sigma)$.

with normal $\mathrm{K}$ giants, where temperature differences of up to $180^{\circ} \mathrm{K}$ occur near $10 \mathrm{~g}$ generally that the greatest precision will be obtained for the dominant component of broadening. If we apply the micro-macroturbulence plus rotation model to $\mathrm{K}$ giant analyses, then macroturbulence dominates, rotation comes next, and microturbulence is smallest. In fact it is even surprising that any information can be obtained on microturbulence in the presence of the two larger broadeners. But we are fortunate in this case because the first zero and the sidelobe structure of the transform are sensitive to the saturation of the line.

If classical depth independent microturbulence of dispersion $\xi$ is introduced into the calculation, it is possible to $\mathrm{fix}$ $\xi$ to within $0.1 \mathrm{~km} / \mathrm{sec}$ (fig. 7) . Larger errors appear in the observed position of the first zero making the errors in $\xi$ more typically $\pm 0.1 \mathrm{~km} / \mathrm{sec}$.

Systematic errors can also come in here because the derived value of $\xi$ depends on $T(\tau)$ and non-LTE effects. In a recent comparison (Gray and Martin 1979) of SMR K giants 
$\tau_{5000}=-3$, the derived values of $\xi$ change by 0.2 to $0.3 \mathrm{~km} / \mathrm{sec}$ which is about 208 of the value of. $\xi$.

The toughest part of the $\mathrm{K}$ giant line broadening analysis though is choosing the ratio $v \sin i / \zeta_{\mathrm{RT}}$ (fig. 8). Errors in this ratio lead to modest errors in $\zeta_{\mathrm{RT}}$, typically $\pm 0.3 \mathrm{~km} / \mathrm{sec}$ (about $7 \%$ of $\zeta_{\mathrm{RT}}$ ) but significantly greater errors in $v \mathrm{sin} i$, typically $\pm 0.5 \mathrm{~km} / \mathrm{sec}$ ( $\geqslant 208$ of $\mathrm{v}$ sin $i$ ). The leverage is good for macroturbulence but poor for rotation. The leverage will be reversed in early type stars where rotational broadening dominates.

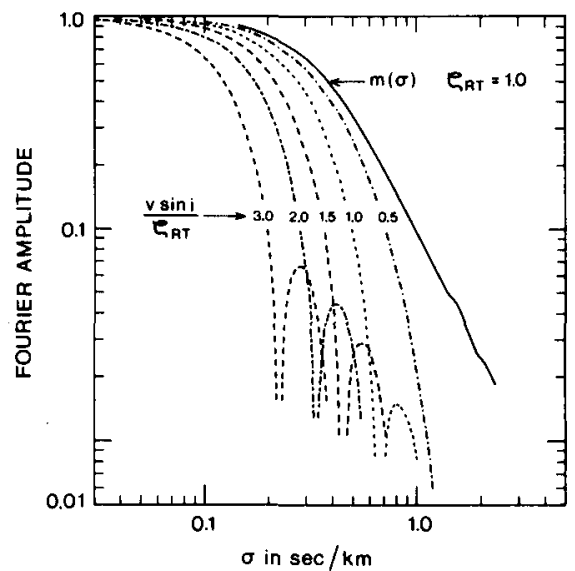

Fig. 8. The separation of macroturbulence from rotation depends on choosing one of these curves from the ensemble on the basis of its shape (from Gray 1977).
Some idea of external consistency can be gained by a comparison of results between workers. For four stars measured by both $M$. Smith and by me, the average deviation from the mean in $\zeta_{\mathrm{RT}}$ was $6 \%$. Rotation values were only consistent to $\sim 35 \%$.

d) Versatility of Transform Analysis

As you can see, the ideas of Fourier transforms are very useful in conceptualizing data collection and analysis. Curiously enough, I received a manuscript not long ago from a competent theoretician in which he spoke of "overcoming" the most serious "flaw" that exists in present Fourier-analysis techniques. By this he apparently meant that he preferred his own models over those used in the past. From my point of view this is an asset not a flaw. We can incorporate new modeling into the Fourier analysis at will. The classical

micro-macroturbulence-rotation model is only one of several possibilities and the value of the Fourier domain is in no way restricted to this model.

The Fourier analysis is particularly suited for discerning small systematic differences in profile curvature where measurements over the whole profile can be brought to bear. (This is exactly the case for macroturbulence vs. rotational broadening.) In some other instances it will be more suitable to use the profile itself.

\section{Analysis in the Wavelength Domain}

It is pretty hard to see the central depth of a line in the Fourier domain. More generally, if we seek source function information, we will want to use the profile itself. I am thinking of the Eddington-Barbier relations and their generalization in terms of contribution functions. We are then forced to live with the uncertainty of 
the noise filter and the observations become tougher.

A typical wavelength domain analysis consists of synthesizing the theoretical line profile and comparing it to the observations. If significant Fourier noise filtering has been made on the data, then the same filter should be applied to the theoretical profile.

But rather than dissipate my remaining time discussing various innuendoes of profile fitting, I will concentrate on one interesting case where profiles tell more than their transforms, namely the measurement of line asymmetries in photospheric lines of late-type stars. One might expect that the imaginary component of the Fourier transform would be the appropriate tool since it is a measure of asymnetry in functions. But it turns out that the imaginary component is too sensitive to small blends and noise in the continuum. Furthermore, the asymetries in these lines involve only a fraction of the whole profile (unlike UV lines in early type stars) which effectively removes one of the leverages of the fourier analysis. Using the profile against its

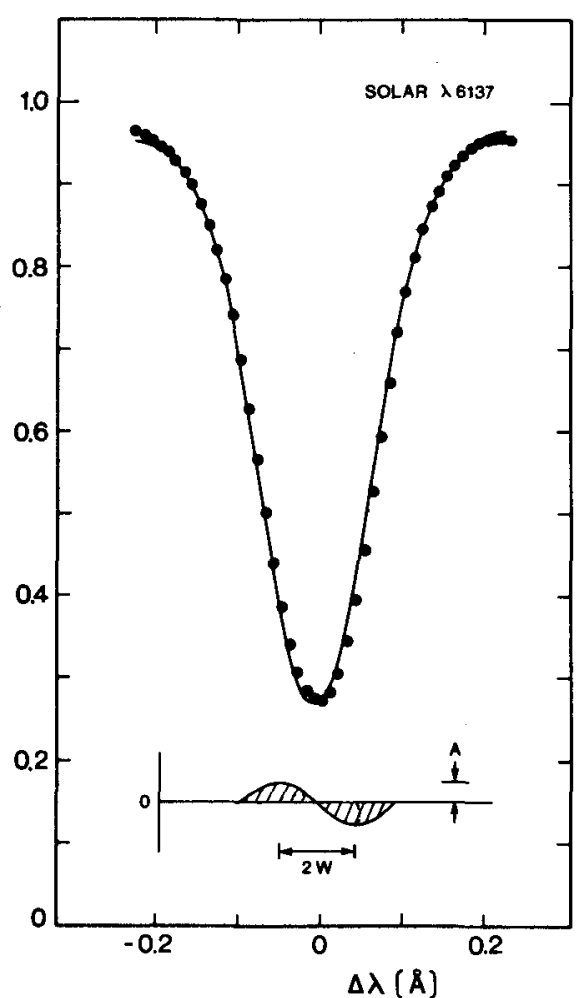

Fig. 9. The asymmetry in solar flux lines shows a red-shifted core.

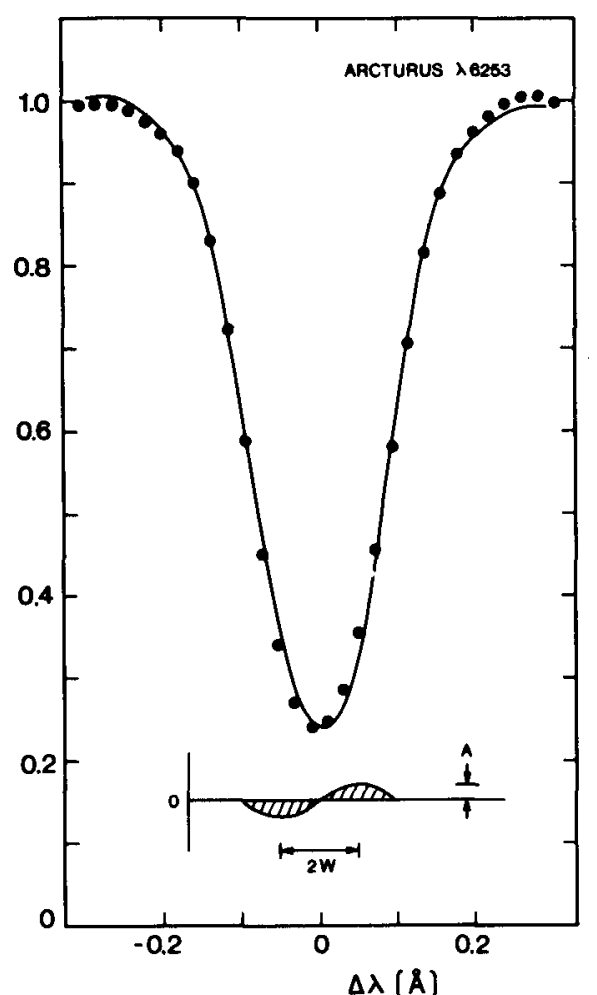

$\Delta \lambda(\AA)$

Fig. 10. The asymmetry in lines of the Arcturus spectrum show blueshifted cores. 
mirror image produces a result like the one in fig. 9. These are solar flux measurements taken from the atlas of Beckers et al. (1976). The process shows very well the familiar red shifted core but neglects the small red shift of the far wings which can be seen only with difficulty.

The traditional measure of asymmetry is the line bisector. It has been used frequently in solar work but is less appropriate in the stellar case. To use it, one needs oversampling of the data and excellent signal to noise. The bisector allows us to compare small sections of the profile with each other. The profile reflection scheme of fig. 9 reduces the subdivision of the profile to the point where we are comparing the core to the wings. The amplitude and width of the difference curve is used to parameterize the asymmetry.

Another example is given in fig. 10 where we see the asymetry in a profile of Arcturus. Here the core is blue shifted.

Notice that the accuracy of the spectrophotometry must be $\sqrt{2}$ times better than the amplitude of the difference curve just to detect the effect. Difference curve ampIitudes are 2-3\%. (Details are being published in the Ap.J.) In addition, one has to worry about spectrograph focus errors and aberrations which can make the instrumental profile asymmetric. These are reasons for placing line asymmetry measurements in toughness category 3 .

5. Summary and Comments

High resolution observations $(\sim 3 \mathrm{~km} / \mathrm{sec})$ with photometric errors of $\lesssim 1 \%$ can be made and are capable of giving physical information about stellar turbulence. There is no one technique for analyzing such data. We can use the advantages of the wavelength domain or the Fourier domain or other transformations yet to be invented according to which of them proves to be the most suitable for the job at hand.

Details of Fourier analysis philosophy and application illustrate the versatility and power in separating line shapes. Uncertainties in modeling remain the biggest obstacle to complete analysis. Adoption of the microturbulence-macroturbulence-rotation model of the broadening allows precision of a few per cent in determining the size of the largest broadener (macroturbulence for late-type stars).

Line asymmetries in late-type stars are more readily measured in the wavelength domain than in the Fourier domain. Exarmles of asymmetries as seen in Arcturus and the solar flux spectrum are given.

We are in a reasonable position for identifying physical mechanisms responsible for stellar line broadening. It is important that theoretical calculations based on various models be explicit in tagging features which are distinct for that model. Then if these features can be observed, they will serve as discriminatns between the physical mechanisms now lumped together under the name turbulence. I am optimistic as I look toward future possibilities. 
Conti, P.S, and Frost, S.A. 1977 Ap.J. $\underline{212,} 728$.

Beckers, J.M., Bridges, C.A., and Gillman, L.B. 1976 Sacramento Peak Observatory Project 7649 , Vols. $\underline{1}, \underline{2}$.

Gray, D.F. 1976 The Observation and Analysis of Stellar Photospheres (New York: wiley).

1977 Ap.J. 218, 530 .

1978 Solar Physics 59, 193.

Gray, D.F. and Martin, B.E. 1979 Ap.J. 231, 139.

Smith, M.A., and Gray, D.F. 1976 P.A.S.P. 88, 809.

Wright, K.O., Lee, E.K., Jacobsen, T.V., Greenstein, J.L. 1964 Publ. Dom. Astrophys. Obs. Victoria 12,173 . 